\title{
Home-based Functional Electrical Stimulation for long-term denervated human muscle: History, basics, results and perspectives of the Vienna Rehabilitation Strategy
}

\author{
Helmut Kern (1,2), Ugo Carraro (1,3) \\ (1) Ludwig Boltzmann Institute of Electrical Stimulation and Physical Rehabilitation, Vienna; \\ (2) Department of Physical Medicine and Rehabilitation, Wilhelminenspital, Vienna, Austria; \\ (3) CIR-Myo Translation Myology Lab, Department of Biomedical Sciences, University of \\ Padova, Italy
}

\begin{abstract}
We will here discuss the following points related to Home-based Functional Electrical Stimulation (h-b FES) as treatment for patients with permanently denervated muscles in their legs: 1. Upper (UMN) and lower motor neuron (LMN) damage to the lower spinal cord; 2. Muscle atrophy/hypertrophy versus processes of degeneration, regeneration, and recovery; 3. Recovery of twitch- and tetanic-contractility by h-b FES; 4. Clinical effects of h-b FES using the protocol of the "Vienna School"; 5. Limitations and perspectives. Arguments in favor of using the Vienna protocol include: 1. Increased muscle size in both legs; 2. Improved tetanic force production after 3-5 months of percutaneous stimulation using long stimulus pulses (> $100 \mathrm{msec}$ ) of high amplitude (> $80 \mathrm{mAmp}$ ), tolerated only in patients with no pain sensibility; 3. Histological and electron microscopic evidence that two years of h-b FES return muscle fibers to a state typical of two weeks denervated muscles with respect to atrophy, disrupted myofibrillar structure, and disorganized Excitation-Contraction Coupling (E-CC) structures; 4. The excitability never recovers to that typical of normal or reinnervated muscles where pulses less than $1 \mathrm{msec}$ in duration and $25 \mathrm{mAmp}$ in intensity excite axons and thereby muscle fibres. It is important to motivate these patients for chronic stimulation throughout life, preferably standing up against the load of the body weight rather than sitting. Only younger and low weight patients can expect to be able to stand-up and do some steps more or less independently. Some patients like to maintain the h-b FES training for decades. Limitations of the procedure are obvious, in part related to the use of multiple, large surface electrodes and the amount of time patients are willing to use for such muscle training.

Key Words: SCI, FES, skeletal muscle permanent long-term denervation, recovery of function, 2D and 3D Color TAC, biopsy, histology, electron microscopy
\end{abstract}

Eur J Trans Myol - Basic Appl Myol 2014; 24 (1): 27-40

The series of e-published issues of the European Journal of Translational Myology (EJTM) are starting in 2014 with the Specials on "The long-term denervated muscle" to resound "The denervated muscle", a book edited in 1962 by one of our virtual mentors, Ernest Gutmann. ${ }^{1}$

To explain why you are now reading this chapter, we need a tremendous amount of details that we cannot include in this review. Indeed, looking to his roots, one of us rediscovered a few months ago (emptying the office for retirement) that his M.D. Thesis was on muscle denervation.

\section{Ugo Carraro: Pioneering studies}

Prof. Carraro would like to remember that in the early 1960s he was a young student at the School of Medicine of the University of Padova, just admitted in 1964 to Internship of the Institute of General Pathology directed by Prof. Massimiliano Aloisi. When in Padua our full Professor almost every day took a tea cup with the fellows, discussing muscle research and his hope to develop in vitro muscle mimics, despite the difficulties to obtain motoneuron-myotubes cultures. Thus, he started lab training doing histology and discussing of the muscle and of its dependence from the motor 


\section{Home-based FES for long-term denervated human muscle}

Eur J Trans Myol - Basic Appl Myol 2014; 24 (1): 27-40

neuron. How to study this topic, if not by denervation experiments? Four years later he defended his M.D. Thesis on: "Impairments of the functions of substructural membranes of the denervated muscle (Alterazioni delle funzioni delle membrane substrutturali nel muscolo denervato). ${ }^{2}$

Now, after 45 years he is trying to convince experts in aging that sparse, but incremental denervation is one of the many mechanisms that worsen muscle performances and quality of life of seniors, and that a long-term high-level physical activity may defer the unavoidable decay of aging., ${ }^{3,4}$ Mosole et al., indeed, comparing muscle biopsies from sedentary and very physically active seniors observed reduced numbers of denervated fibers and higher percentages of throphic and slow-type groups of reinnervated fibers in the active group. The observations suggest that long-term physical activity promotes reinnervation of muscle fibers undergoing age-related denervation.

Here he may only list the topics he would like to describe in a future book. He will need to start with his mentors (Aloisi, Zatti and Margreth), and describe the importance for his ability to design and perform independent research of his younger or older colleagues Catani, Mussini, Cantini, Salviati and Schiaffino. The explanation of why in Padua there was and there is such a strong tradition of Myology will end the first chapter. It will be a funny story related to fever and burning of toxins in the muscle ...

He will explain why he moved from General Pathology to Muscle Biology and Physiopathology, from Basic to Applied Myology, organizing the Interdepartmental Research Center of Myology of the University of Padova, in which clinical colleages and biomedical scientists are almost equally present, from organizing the PaduaMuscleDays Meetings and editing the journal Basic and Applied Myology (BAM) to the European Journal of Translational Myology.

He will mention the inter-relationships among his students (Donatella Biral, Donatella Morale, Giorgio Vescovo, Corrado Rizzi, Gianluca Rigatelli, Marco Sandri, Marzena Podhorska-Okolov, Katia Rossini, Massimo Donà, Nicoletta Adami, Sandra Zampieri and Simone Mosole), the visits and lab periods spent in international laboratories (in particular those of John Gergely and Alfred Goldberg in Boston), the Italian and International friends with which he has published papers (Anna Jakubiec-Puka, Claudio Franceschi, Giorgio Arpesella, Mike V. Dodson, Stanley Salmons, Winfried Mayr, Simona Boncompagni, Feliciano Protasi, Antonio Musarò, Giorgio Fanò, Vincenzo Vindigni, Franco Bassetto, Francesco Mazzoleni, Dan Graupe, Amber Pond, Marina Marini, Fabio Francini, Paolo Gargiulo, Thordur Helgason, Tiziana Pietrangelo, Nejc Sarabon, and last but not least Helmut Kern) and of course the many others he met during International Conferences.
His "first" Meeting, as a young fellow of myology was organized in Switzerland by Marcus C. Schaub; but how to forget the International Conferences where he and his students had the chance to know Bruce $\mathrm{M}$. Carlson, John Faulkner, Zipora Yablonka-Reuveni, Eric Monnet, Miranda Grounds, Winfried Mayr and many other Vienna friends) or the Conference he organized in Terme Euganee, Padua: where we met Juan Carlos Chachques, a young Surgeon from Argentina working in Paris with Alain Carpentier, Carlo Reggiani, now full professor of Physiology in Padua University, Terje Lomo, Dirk Pette, Salvatore Di Mauro, Clara Franzini-Armstrong, Tessa Gordon, Victor Dubowitz, Terry Partridge, Ryoichi Matsuda, Stanley Salmons, Jonathan C. Jarvis, Dario Coletti, Werner Lindenthaler and many others. Of Gerta Vrbova he will remember that she was one of the first invited speakers he personally met in 1979 in the Margreth's Lab, as a young fellow who presented to her his first independent publication on "selective maintenance of neurotrophically regulated proteins in long-term denervated hemidiaphragm". 5 Finally, he will identify the main research topics he worked on during 45 years of research activity. He started in 1966 to prepare the M.D. Thesis, but he is not yet ready "retire" from Myology: as a Senior Scholar of the University of Padova, he think that he has a lot to translate to clinical colleagues.

The first topic of his long career was: Contractile protein isoforms identified by several electrophoretic methods ${ }^{6,7}$ and their transitions as tools to study modulation and pathology of muscle fiber units and motoneurons. He will remember his first publication on "Neural control on the activity of the calciumtransport system in sarcoplasmic reticulum of rat skeletal muscle" by Margreth, Salviati, Carraro in Nature $1973^{8}$ and six years later "denervation-induced isomyosin transitions" by Carraro, Catani, Biral. Exp Neurol $1979^{5}$ and by Carraro et al. $1985 .^{9}$ Some years afterwards, inspired by Terje Lomo and Stefano Schiaffino, ${ }^{10-19}$ he independently did corroborating experiments of continuous electrical stimulation of denervated muscle, achieving a yet unexplained and infrequently cited high increase of slow muscle fibers properties in denervated fast muscle. ${ }^{20} \mathrm{~A}$ system analysis with flow charts may summarizes all the interactions among old and recent topics, Carraro's mentors - students - Padua colleagues with Italian and International Scientists/Clinicians involved in animal and human muscle biology, pathology, therapy and rehabilitation, but he belive he will need months if not years to complete his book project.

He has to describe: Muscle damage and regeneration via myoblast's proliferation, differentiation and fusion, ${ }^{21,22}$ including exercise-induced muscle fiber apoptosis in normal and dystrophic animal and human muscles. $^{23,24} \mathrm{He}$ studied: Isomyosins in hypertension and heart failure ${ }^{25}$ and introduced the concept of 


\section{Home-based FES for long-term denervated human muscle}

Eur J Trans Myol - Basic Appl Myol 2014; 24 (1): 27-40

"Demand Dynamic Cardio-myoplasty," first in a sheep model with Giorgio Arpesella ${ }^{26}$ and then in patients with Gianluca Rigatelli. ${ }^{27}$ Corroborating evidence of effectiveness of the intermittent stimulation strategy was collected on other sheep models. ${ }^{28,29}$

Finally, the strong leadership of Helmut Kern convinced Engineers in Vienna and then myologists in Italy (Carraro's team in Padua and Feliciano Protasi in Chieti) to implement two pilot trials supported by the EU Project RISE, the first a cross-sectional study ${ }^{30-36}$ and then a longitudinal-study ${ }^{37-40}$ to demonstrate that a home-based strategy of Functional Electrical Stimulation (h-b FES) recovers muscle mass and functions of permanently denervated human muscle.

With EU-support, Helmut Kern and his European collaborators, Carraro's team included, are now translating this strategy to the more frequent cases of muscle deterioration due to aging and cancer. ${ }^{3,41-43}$

Further, in Padua he is extending the EU RISE results to partially reinnervating muscle, developing dedicated monitoring strategies. ${ }^{39,44,45}$ To objectivize results of these researches, he is proud to have revitalized the clinical use of ultrasound muscle approaches, adding dynamic analyses of contractile properties in clinical evaluation of denervated and reinnervating muscles. Further, he suggested to Paolo Gargiulo and Helmut Kern to add false color to "Monitoring of muscle and bone recovery in spinal cord injury using threedimensional imaging and segmentation techniques", to allow doctors and their patients to read much easy-tointerpret Computer Tomography analyses of their deteriorating or recovering muscles. ${ }^{38,39,46}$

That is why after almost 50 years, he is still fond of the effects of denervation and of the modulation by electrical stimulation of skeletal muscle fibers, of their adaptation/damage/apoptosis/regeneration potentials by reciprocal interactions with inflammatory cells and nerve, hoping to identify further clues worth to be translated into clinically relevant therapy and rehabilitation strategies.

\section{Helmut Kern: Pioneering research}

In 1990 dr. Helmut Kern achieved his Habilitation for M.D. with a thesis that has been published in German in the Oesterreichische Zeitschrift fuer Physikalische Medizin 1995; 5: Heft 1, Supplementum. ${ }^{47}$

The thesis is now reprinted in the special issues "The long-term denervated muscle" ${ }^{48}$ The English abstract is provided in the following paragraph.

\section{Functional Electrical Stimulation on Paraplegic} Patients.

We report on clinical and physiological effects of 8 months Functional Electrical Stimulation (FES) of quadriceps femoris muscle on 16 paraplegic patients. Each patient had muscle biopsies, CT-muscle diameter measurements, knee extension strength testing carried out before and after 8 months FES training. Skin perfusion was documented through infrared telethermography and xenon clearance, muscle perfusion was recorded through thallium scintigraphy. After 8 months FES training baseline skin perfusion showed $86 \%$ increase, muscle perfusion was augmented by $87 \%$. Muscle fiber diameters showed an average increase of $59 \%$ after 8 months FES training. Muscles in patients with spastic paresis as well as in patients with denervation showed an increase in aerob and anaerob muscle enzymes up to the normal range. Even without axonal neurotropic substances FES was able to demonstrate fiber hypertrophy, enzyme adaptation and intracellular structural benefits in denervated muscles. The increment in muscle area as visible on CT-scans of quadriceps femoris was $30 \%$ in spastic paraplegia and $10 \%$ in denervated patients respectively. FES induced changes were less in areas not directly underneath the surface electrodes. We strongly recommend the use of Kern's current for FES in denervated muscles to induce tetanic muscle contractions as we formed a very critical opinion of conventional exponential current. In patients with conus-cauda-lesions FES must be integrated into modern rehabilitation to prevent extreme muscle degeneration and decubitus ulcers. Using FES we are able to improve metabolism and induce positive trophic changes in our patients' lower extremities. In spastic paraplegics the functions , rising and walking“ achieved through FES are much better training than FES ergometers. Larger muscle masses are activated and an increased heart rate is measured, therefore the impact on cardiovascular fitness and metabolism is much greater. This effectively addresses and prevents all problems which result from inactivity in paraplegic patients.

The 325 references are a remarkable collection of the pioneering work on FES in paraplegics that ended up with the first world implant of a device performed in Vienna in $1983 .{ }^{49}$

Since then, an enormous amount of new work has been necessary to establish a clinically accepted strategy for recovery of contractile function of long-term denervated muscle, but the work in the 1970's and 1980 's has provided a firm and accurate basis for the current understanding of the recovery process in human muscles.

\section{Collaboration of Austrian and Italian researchers}

In 1998 Helmut Kern went once again in Terme Euganee to cycle on the Euganei Hills. A late morning he went to the Padua Institute of General Pathology with his Habilitation Thesis to meet Ugo Carraro and to express is strong willingness to collaborate in a scientific study of a series of Conus Cauda sufferers he was training with h-b FES since several years. The reply of Ugo was outspoken: "Helmut harvest a muscle 


\section{Home-based FES for long-term denervated human muscle}

Eur J Trans Myol - Basic Appl Myol 2014; 24 (1): 27-40

biopsy and we will show to skeptics that the astonishing functional improvements in muscle contractility you achieved with your elegant training strategy will be supported by evidence of improved muscle fiber size and ultrastructural features". The first biopsy from muscles treated in this way is described in two articles that report the characteristics of the muscle fibers from the Quadriceps of a person after 26 months of denervation and h-b FES Training. ${ }^{30,32}$

From the second article, submitted years before acceptance (and only after a cross-sectional study published in a prestigious journal provided stronger evidence of the effectiveness of the $h-b$ FES for denervated muscles ${ }^{32}$ ) we here republish the clinical description of this first, very successful case.

"V. Z., a 47-year-old man, had suffered a traumatic cauda equina lesion at T12. One year later, his quadriceps femoris muscles were severely wasted on both sides. Voluntary movement, sensation, and reflexes were all absent, consistent with total denervation. After a further 6 months, findings at neurological examination were unchanged. Absence of volitional activity on needle electromyography (EMG) and of evoked activity using surface EMG with transcranial and lumbosacral magnetic stimulation confirmed permanent and complete loss of motor functions of spinal nerves L1 to L4. Direct electrical stimulation, which in a normal muscle would elicit a response with a chronaxie of 0.1-0.7 $\mathrm{ms}$, required a chronaxie of more than $20 \mathrm{~ms}$, constituting further evidence of complete loss of innervation. A computerized tomography $(C T)$ scan of the thighs revealed marked atrophy of muscle tissue with replacement by fat; the cross-sectional areas of the quadriceps muscles were $36.0 \mathrm{~cm} 2$ (right) and 36.1 cm2 (left, Fig. 1A), representing 58.9\% (right) and $59.1 \%$ (left) of the corresponding areas in a typical healthy individual. No detectable knee extension torque could be elicited by stimulation under isometric conditions with the subject sitting with the knee flexed at $90^{\circ}$. Eighteen months after his injury, V. Z. commenced a training program which, after appropriate instruction, he was able to carry out at home. Two pairs of large electrodes, each having an area of $200 \mathrm{~cm} 2$, were strapped to the anterior surface of the thighs in proximal and distal positions. Twitch contractions were elicited by biphasic rectangular current pulses having duration of $120 \mathrm{~ms}$ and amplitude of $200 \mathrm{~mA}$, delivering impulse energy of 1.92 Joules, to recruit fibers throughout the quadriceps femoris muscles. The long duration of the impulses needed for stimulation precluded the use of frequencies that would elicit tetanic contractions; training was therefore initiated with single twitches at $2 \mathrm{~Hz}$ and delivered for 15 min per day, 5 days per week. After 4 months, excitability of the muscle fibers had recovered sufficiently for pulses of shorter duration to be used. At this stage, the protocol was augmented with an additional tetanic pattern consisting of pulses of $40 \mathrm{~ms}$ delivered at $20 \mathrm{~Hz}$ for $2 \mathrm{~s}$ on, $2 \mathrm{~s}$ off for 15 min daily, 5 days per week. The total amount of stimulation was then $30 \mathrm{~min}$ daily for each muscle. The additional tetanic stimulation pattern produced more rapid and more forceful contractions, resulting in a progressive increase in knee extension torque. After 26 months of stimulation $V$. Z.'s thighs came to resemble those of a healthy sedentary subject; although the external appearance was not entirely normal, it was certainly more acceptable cosmetically to the patient. CT scan showed that the cross-sectional areas of the quadriceps muscles at the same level had increased on the right side from 36.0 to $57.9 \mathrm{~cm} 2$ and on the left side from 36.1 to $52.4 \mathrm{~cm} 2$ (Fig. 1B); these figures represent $94.7 \%$ (right) and $85.7 \%$ (left) of the areas typical of a healthy subject. Muscle density, expressed in Hounsfield Units, had risen from 11.0 to 26.4 on the right side and from 10.7 to 24.1 on the left. Stimulation of the quadriceps muscles elicited a knee extension torque of $12.0 \mathrm{Nm}$ on the right and $10.5 \mathrm{Nm}$ on the left. Despite the marked restoration of muscle cross-sectional area, this was less than $10 \%$ that of a normal subject. Nevertheless, this stimulation-induced torque enabled $V$. Z. to extend the knee from a sitting position and to maintain a standing posture without the support of the upper extremities.

Biopsies were taken from the right and left vastus lateralis muscles and frozen sections were stained with hematoxylin and eosin and with a monoclonal antibody (NCL-MHCd; Novocastra Laboratories Ltd, Newcastle upon Tyne, United Kingdom) to the embryonic myosin heavy chain isoform (MHCemb).The sections consisted mainly of large round myofibers with a mean diameter of 37.2 +/- $24.8 \mu \mathrm{m}$ (right) and 40.5 +/- $24.9 \mu \mathrm{m}$ (left). There was very little fat or fibrous connective tissue. Small myofibers (<10 um diameter) were also present. Some appeared to be severely atrophic (Fig. 1C, arrowheads); others, intensely basophilic with several large internal nuclei, we interpret as undergoing regeneration (Fig. 1C, arrows). The latter stained positively with anti-MHCemb (Fig. 1D, green coloration), providing evidence of their recent formation. The antibody also reacted with some larger myofibers (> $30 \mu \mathrm{m}$ diameter) with subsarcolemmal myonuclei; we have seen similar fibers in permanently denervated rat muscles in which regeneration had been induced by myotoxin treatment. MHCemb-positive myofibers constituted $8.7 \%$ (right) and $2.3 \%$ (left) of the identifiable muscle fibers present in the biopsies.

Paralysis and denervation were demonstrated clinically in this patient at 12 months and 18 months post-injury and again after 26 months of stimulation. We conclude that the injury was stable and that no recovery could have occurred spontaneously during the period of treatment. Nonetheless, the intensive 


\section{Home-based FES for long-term denervated human muscle}

Eur J Trans Myol - Basic Appl Myol 2014; 24 (1): 27-40

regime of electrical stimulation was associated with an increase in excitability, size, and force-generating capacity of the denervated quadriceps muscles, and this was sufficient to allow knee extension to be induced by electrical stimulation. There was histologic evidence of an accompanying reduction in fat and connective tissue, of growth in diameter of surviving myofibers, and also of regenerative phenomena resulting in the formation of new myofibers.

Previous studies on denervated muscles in both animals and humans have shown that electrical stimulation can induce a small increase in muscle mass and hypertrophy of the denervated muscle fibers. The present case is unusual in the extent of the changes produced in the long-term denervated and stimulated muscles, and in the evidence it provides for the participation of both hypertrophy and hyperplasia in the recovery process."

\section{The multi-stage response of human muscle fibers to long-lasting denervation}

The complex response of muscle tissue to denervation is one of the most studied processes in muscle physiology and pathology. One of the important conclusions of pioneering studies ${ }^{1}$ is that after a certain period of time (seven months in the rat, see Bruce M. Carlson's review in this issue $e^{50}$ ), denervated muscle undergoes some irreversible changes that inhibit its full restoration even after reinnervation. Findings from different experimental models, including free autotransplant, led to the same conclusion: rat muscle denervated up to two months is restored as well as grafts of control muscles, but between two and seven months of denervation, the restorative capacity declines progressively before leveling out at a very low level at seven months of denervation. ${ }^{51}$ These observations were translated to macroscopic behavior of denervated human muscle and its potential for reinnervation, establishing a dogma (muscle fibers are fully lost six months after denervation) that continues to influence clinical managements of human muscle denervation.

Despite countless published results, our recent studies in animal models and humans are enlightening several unrecognized characteristics and behaviors of the complex processes that occur during permanent LMN denervation of the muscle tissue. These results are strengthening the rational basis of $\mathrm{h}-\mathrm{b}$ FES to maintain/recover permanently LMN denervated muscles. Some of the effects of long-lasting (in terms of years) LMN denervation of human muscle we recently described were unexpected. In contrast with the well-known rodent model, one year after SCI the LMN denervated human muscle presents simple atrophy. ${ }^{31}$ These results extend preliminary observation obtained from the analyses of muscle biopsies in a prospective study on human free flap muscle transfers, which showed that at nine months follow-up after surgery the denervated muscle fibers only decreased in size. ${ }^{52}$ On the other hand, characteristic denervationinduced muscle fiber disorganization is documented by electron microscopy in the atrophying muscle. ${ }^{31,34} \mathrm{We}$ would like to stress that ultra structural disorganization of the muscle fibers appears much earlier than severe atrophy in both animal models, ${ }^{53}$ and humans, ${ }^{31,44}$ thus explaining the early functional impairments of the LMN denervated muscle.

At light microscopy level, a severe tissue degeneration in humans starts only during the second year of SCI, fibro-fatty muscle substitution being accompanied by other cellular processes: i) countless severely atrophic muscle fibers with nuclear clumpings, ${ }^{54}$ ii) "swollen" fast-type muscle fibers; and iii) myoblast proliferationdependent regenerated muscle fiber [myotubes, neural cell adhesion molecule (N-CAM) and embryonic myosin heavy chain (MHCemb) positive "young" fibers and large onion-like muscle fibers]. In permanently denervated human muscles between the third and fifth year post SCI, severely atrophic fibers with nuclear reorganization fill the loose connective tissue. These severely atrophic muscle fibers lose completely their myofibrillar apparatus and the coil distribution of myonuclei that are relocated in groups (nuclear clumps) in the center of the muscle fibers. ${ }^{54}$

Satellite cell activation, replication and fusion to regenerating muscle fibers occur from the first to at least the tenth year of LMN denervation. Indeed, in the human denervated muscle around $2 \%$ of the residual muscle fibers express, transiently, MHCemb, as part of the processes of non-compensatory myogenesis. ${ }^{31,33}$ This phenomenon has also been demonstrated in rodents after a year-long permanent denervation in both hemidiaphragm and leg muscles ${ }^{9,55}$ and then confirmed by Kern et al. in humans up to 20 years after LMN denervation. ${ }^{30,31}$ both without and with h-b FES, which effectively reverted long-term atrophy and conceivably maintained the mass of regenerated myofibers. ${ }^{31}$ Regeneration of muscle fibers in human LMN denervated muscle has also been demonstrated in muscle biopsies harvested from free muscle transfers up to four years after surgery, where activation of satellite cells and MHCemb-positive muscle fibers were evident. ${ }^{52}$ Besides regenerating myotubes and small round muscle fibers, in long-lasting denervation, large onion-like muscle fibers are also frequent. ${ }^{31}$ These myopathic features are the results of the incomplete fusion of myoblasts during aneural regenerative events. This has been demonstrated to produce several branched myofibers, when seen in longitudinal section, inside the remaining basal membrane of a dead myofiber in rodents. ${ }^{9}$ Strong corroborating evidence was collected analyzing expression of myogenesis-related genes in denervated rat muscles. ${ }^{56,57}$ Thus, it can be concluded that human muscle fibers survive permanent LMN denervation much longer than generally accepted, providing the 


\section{Home-based FES for long-term denervated human muscle}

Eur J Trans Myol - Basic Appl Myol 2014; 24 (1): 27-40

rationale to plan research aimed to recover long-lasting denervated muscle.

Permanent denervation of the limbs muscles due to LMN lesion may take place when trauma to spinal cord, roots and peripheral nerves occurs. Examples are spinal cord lesion with concomitant root damage, brachial plexus palsy and sciatic nerve injury. When proximal denervation occurs, it may be necessary more than a year for reinnervation, during which the process of severe atrophy and fibrosis of the affected muscle tissue may impair synaptic reorganization.

One of the effects of SCI is the rapid loss of contractile force and mass of the affected muscles. Atrophy of leg muscles is particularly severe when the injury destroys the LMN and, hence, the contacts between motor neurons and muscle fibers. In this case, within weeks atrophying and fibrillating muscles become unable to sustain tension during tetanic contractions induced by electrical stimulation. Within months, the denervated leg muscles are no longer excitable by standard commercial electrical stimulators because they have undergone severe disorganization of contractile elements (myofibrils) and of the excitation-contraction coupling apparatuses. ${ }^{31}$ Finally, after years of denervation, muscle fibers are replaced with adipose and fibrous tissues. ${ }^{30,31,37,38,58}$

These severe functional and structural changes of denervated muscle tissue are not detectable in patients suffering with complete upper motor neuron (UMN) lesions even 20 years after SCI at thoracic level. ${ }^{59}$ On the other hand, larger trauma of the lumbar and ischiatic regions, complicated by ischemic and infection necrosis of the spinal cord, may extend the damage to large segments of the medulla and of the nerve roots. In these latter cases, the diagnostic problems are related to completeness of the LMN denervation, while the absence of sensation of the legs and of the pelvic sphincters grants completeness of the transverse spinal cord lesion (ASIA grade A of SCI).

To avoid problems in interpreting clinical results related to residual LMN innervation or reinnervation, we firstly designed and implemented a cross-sectional study $^{31}$ followed by a longitudinal 2-year long prospective study $^{37,38}$ that recruited 25 paraplegic patients specifically selected because of complete LMN denervation of the quadriceps muscles. In the longitudinal study, the same group of patients was assessed before and after two years of h-b FES by clinical, functional, imaging and muscle biopsy analyses. ${ }^{37,38,60}$ To avoid the potential criticisms related to a possible residual innervation or reinnervation, dedicated diagnostic protocols were designed and implemented to test "completeness" of LMN denervation of right and left quadriceps muscles before and during the two years of the study. ${ }^{38,61}$ By means of these tests, in particular Test electrical stimulation by bidirectional rectangular impulses of $1 \mathrm{~ms}, 40 \mathrm{~Hz}$, $100 \mathrm{~mA}$ of amplitude testing for contraction of the thigh muscle, the complete and permanent denervation of the quadriceps before the beginning of h-b FES, and after two years of training was fully granted, since the stimulated muscle improved their excitability recovering tetanic contractility, but never responded to the settings which are able to elicit contraction of innervated muscle. ${ }^{38}$ If no electrical stimulators providing the high level stimulation parameters are available, electrical stimulation with bidirectional rectangular impulses of about $1 \mathrm{~ms}$, a frequency of $40 \mathrm{~Hz}$ and an intensity of $100 \mathrm{~mA}$ can be used for the first evaluation of the paralyzed muscle. These parameters can be delivered by most of the commercially available devices and are sufficient for a first diagnosis if the stimulated muscle shows signs of denervation. All together, the above described behaviors leave a significant time window for intervention to avoid degeneration of the LMN denervated muscle by home based electrical stimulation.

From the first biopsy to the end of the European Project RISE: Use of electrical stimulation to restore standing in paraplegics with long-term denervated degenerated muscles (Contract no. QLG5-CT-2001-02191)

From early 2000 to August 2004 more than 130 biopsies of Conus Cauda Patients were analyzed in Padua (by morphometry and immunostaining) and in Chieti (electron microscopy). Further, muscle biopsies from spastic paraplegics (i.e., those with lesion of the upper motoneuron) were also analyzed to described the differential behaviors of truly disconnected muscle fibers to those severely atrophic (but never degenerated) due to severe unloading. ${ }^{59}$

Aim of the EU Commission Shared Cost Project RISE (Contract no. QLG5-CT-2001-02191) was to confirm previous results of a cross-sectional study $^{31}$ by a longitudinal prospective study in 25 paraplegic patients specifically selected because of complete LMN denervation of the quadriceps muscles. The overall conclusions, taken from Kern et al. 2010 NNR $\operatorname{article}^{38}$, of all these studies may be summarized as follows:

"Atrophy of skeletal muscle groups is particularly severe when SCI involves all the lower motor neurons (LMNs). After such a complete injury, the peripheral endings of motor neurons quickly degenerate whereas LMN denervated muscles undergo progressive decay, which can be roughly divided in the following chronological steps: (a) in days denervated muscle starts to spontaneously activate action potentials (fibrillations); (b) in weeks, muscles become unable to sustain tension during tetanic contractions induced by electrical stimulation; (c) within months, muscles are unexcitable with standard commercial electrical stimulators,[62-66] undergoing ultrastructural severe 


\section{Home-based FES for long-term denervated human muscle}

Eur J Trans Myol - Basic Appl Myol 2014; 24 (1): 27-40

disorganization of the E-C Coupling and of the contractile apparatuses; and (d) after years, the myofibers are replaced by adipocytes and collagen.

To counteract the progressive changes that transform muscle into an unexcitable tissue, over the past 20 years we have developed a novel therapy concept for paraplegic patients with bilateral and complete LMN denervation of the lower extremity due to complete lesions of the conus and cauda equina (CC). This new training strategy became possible because of the development of a new generation of stimulation equipment specifically designed for home-based functional electrical stimulation ( $h-b$ FES). These new stimulators and the large surface electrodes necessary to cover the denervated muscles were developed by the Center of Biomedical Engineering and Physics at the Medical University of Vienna and by the Wilhelminenspital, Vienna (Austria), to reverse longstanding and severe atrophy by delivering highintensity and long-duration impulses that can directly elicit contraction of denervated skeletal fibers in the absence of nerve endings. Our data, indeed, show that $h-b$ FES can be an effective home therapy to counteract muscle atrophy and degeneration after complete LMN denervation due to CC lesions. The $h-b$ FES device stimulates muscle fibers in the absence of nerve endings and after prolonged denervation, enabling (a) recovery of muscle mass and fiber size, (b) recovery of tetanic contractility, and (c) restoration of muscle fiber ultrastructure.

Up to now, the muscles of affected extremities in these paraplegic patients are commonly not treated with FES because it is widely accepted that long-term and completely denervated muscles cannot be effectively stimulated. On the other hand, studies in animal models and humans indicate that (a) severe atrophy does not occur in rats for at least 3 to 4 months; (c) in rabbit, the degeneration of muscle tissue does not appear during the first year of denervation; and (c) in humans, muscle tissue degeneration starts from the third year onward. Our recent findings that the longterm denervated rat muscle maintains L-type Ca2+ current and gene expression of the related proteins longer than functional contractile machinery provide the molecular, structural, and functional rationale of rehabilitation training for permanently denervated muscles, consistent with clinical observations. This leaves a window of opportunity to initiate muscle stimulation and avoid muscle degeneration and infiltration.

Our light microscopy results suggest a window for intervention in patients up to 2 years after injury, because fibers maintain at least 30\% of their initial size and the extracellular matrix is still evolving. EM analyses, on the other hand, indicate that the structure of the sarcotubular system (reputed to deliver action potential to the fiber interior) and myofibrils decays quite quickly, suggesting that it is best to start h-b FES training as soon as possible after SCI, possibly not later than six months. The poor excitability/contractility of human long-term denervated fibers is likely attributable to ultrastructural changes that affect the EC coupling apparatus and contractile elements and precede severe atrophy and degeneration. The reorganization of $T$ tubules and $\mathrm{Ca} 2+$ release units and myofibrils that follows $h$ - $b$ FES likely plays a role in the recovered ability of LMN denervated muscles to be stimulated and to respond with tetanic contractions.

Because the progression of recovery in $h-b$ FEStrained LMN denervated muscle is inherently slow, in part due to exercise training for only 30 minutes per muscle group, 5 times a week, patients were clinically evaluated every 12 weeks by physiatrists, who progressively modified the stimulation parameters and training protocol according to the patient's improvements. During the first few months of $h-b$ FES training, the initially poor excitability of the denervated muscle was improved by twitch-contraction training. Three to 6 months later, electrical stimulation induced tetanic contractions against loads that were progressively increased, accompanied by a significant increase in the mass of the quadriceps muscles (24\% at the midterm evaluation) and by improvement in limb appearance and muscle cushioning. None of the subjects that reached 1 year $h$ - $b$ FES training $(n=20)$ declined in terms of their muscle properties, and $20 \%$ reached the ability to perform stand-up exercise assisted by electrical stimulation of quadriceps muscles.

At 2 years, $90 \%(n=20)$ of $h-b$ FES trained subjects recovered or increased tetanic contractions, and $25 \%$ stood during electrical stimulation in parallel bars. Minimal functional improvements were associated with long time elapses between SCI and initiation of $h-b$ FES and possibly lower compliance with training. In single case reports, low compliance substantially decreased the effects of training, yet in the same subjects the mass of thigh muscles increased when the patient resumed $h-b$ FES.

The likelihood that the lower extremities of these patients were completely denervated before initiation of $h$ - $b$ FES training and remained denervated during and after the 2 years of training was indicated by several assessments (e.g., test electrical stimulation, needle electromyography, transcranial and lumbosacral magnetic stimulation). In particular, the threshold of excitability of the quadriceps muscles never increased to a level that allowed them to respond to standard commercial electrical stimulators (impulse duration about 0.5-2 ms), which elicit a muscle contraction through the nerve. The severity of postdenervation atrophy (and the extent of $h-b$ FESinduced recovery) was similar in the left and right quadriceps muscles of the same patient. In incomplete denervation (or some re-innervation), we would have 


\section{Home-based FES for long-term denervated human muscle}

Eur J Trans Myol - Basic Appl Myol 2014; 24 (1): 27-40

expected greater variability. Finally, patients did not describe pain during surface stimulation with high current (1000-3000 times higher energy [2.4 J at 120 $\mathrm{ms}$ and $200 \mathrm{~mA}$ ] than that delivered by standard commercial stimulators [0.8 $\mathrm{mJ}$ at $0.7 \mathrm{~ms}$ and $50 \mathrm{~mA}]$ ), implying complete sensory loss.

In conclusion, our findings strongly support the RISE rehabilitation protocol as a method to improve the mass and contractility of LMN denervated muscles, although we found a limited "measurable" knee torque changes in h-b FES trained muscles. These benefits could be extended to patients with similar lesions, especially to determine whether $h-b$ FES can reduce secondary complications related to disuse and impaired blood perfusion (reduction in bone density, risk of bone fracture, decubitus ulcers, and pulmonary thromboembolism).

On the other hand, the Authors share the following suggestions of Gerta Vrbova, which was so kind to attract our attention on the intrinsic limiting factors that will never allow long term denervated muscle to reach by Electrical Stimulation (as it is feasible in clinical settings) the stage of a fully normal muscle. Indeed, our main evidence for muscle denervation even after years of h-b FES is the fact that the trained muscles never attain the ability to respond to the much lower currents that stimulate curarized or the denervated muscle fibers early after degeneration of the peripheral nerve stump.

"While there is no doubt that impulse activity has a decisive role in determining muscle properties [67] it cannot entire replace the effect of innervation on denervated muscle. Whether this is due to a trophic influence of nerve on muscle or other factors has not yet been resolved.

There are several possible reasons why electrical stimulation cannot entirely mimic the effect of innervation on skeletal muscle: 1. Denervated muscles are stimulated in a manner that causes synchronous contraction of all muscle fibers in the stimulated muscle. This differs greatly from the activity that the nerve is imposing onto the muscle it innervates. During nerve induced movement different motor units are activated asynchronously, and never at the same time [68]. Thus the recruitment order of different muscle fibers is completely different from electrically induced muscle stimulation. 2. The synchronous activity of denervated muscles cannot mimic that which occurs during natural movement and as a consequence the mechanical conditions of different muscle fibers within the stimulated muscle will be far from normal. The amount of load during contraction affects slow muscle fibers more than fast ones; indeed they degenerate if they contract in the absence of load [69]. 3. The simple interpretation of the effect of whole muscle stimulation is therefore limited for synchronous stimulation of all muscle fibers in denervated muscles is very different from nerve induced activity during normal movement. 4. Apart from the superbly organized recruitment order of motor units during normal movement that seems to be necessary for the integrity of the different types of muscle fibers there could be an additional trophic effect of the nerve on muscle but there is little evidence for such an influence that is independent on muscle activity or the mechanical conditions.

We are aware, indeed, that the clinical results may appear poor or very poor to "normal people", but, please, reader consider them from the point of view of a disabled person at risk of serious complications. The increase in mass (cushioning effect) and the antigravitational pumping of leg blood are muscle "functions" that are fully lost after denervation, but are substantially recovered during long-term daily electrical stimulation.

\section{Devices and Vienna Stimulation Strategy for $h-b$ FES of large denervated human muscles in SCI}

To counteract the progressive changes that transform muscle into an unexcitable tissue unable to generate force with standard commercial stimulators (from six months onward), in the past 20 years Clinicians and Engineers developed in Vienna novel rehabilitation concepts for paraplegic patients with bilateral and complete LMN denervation of the lower extremity due to complete lesion of the Conus Cauda ${ }^{70}$ This new rehabilitation protocol became possible due to the development and optimization of new stimulation equipments for FES. The devices have been specifically designed to reverse longstanding and severe atrophy of LMN denervated muscles by delivering high-intensity and long-duration impulses that can directly elicit contraction of denervated skeletal fibers in absence of nerve endings. These new stimulators and the large surface electrodes needed to cover the denervated muscles were developed by the Center of Biomedical Engineering and Physics at the Medical University of Vienna, Austria. ${ }^{71-73}$ In parallel, specific clinical assessments and training settings were developed at the Wilhelminenspital Wien, Austria. $61,74,75$ The rehabilitation progressive training strategy for LMN denervated muscles (see the Figure 1) are validated by the clinical results, strongly supported by those obtained from light and electron microscopy muscle biopsies' analyses performed in Padua and Chieti Universities (Italy), respectively, as described by Kern et al. in the longitudinal prospective study. ${ }^{38,59}$ Patients were provided with stimulators and electrodes in order to perform stimulation at home for five days per week. The large $(180 \mathrm{~cm} 2)$ electrodes (Schuhfried GmbH, Mödling, Austria) made of conductive polyurethane, were placed on the skin surface using a wet sponge cloth (early training) and fixed via elastic textile cuffs. As soon as the skin was accustomed to 

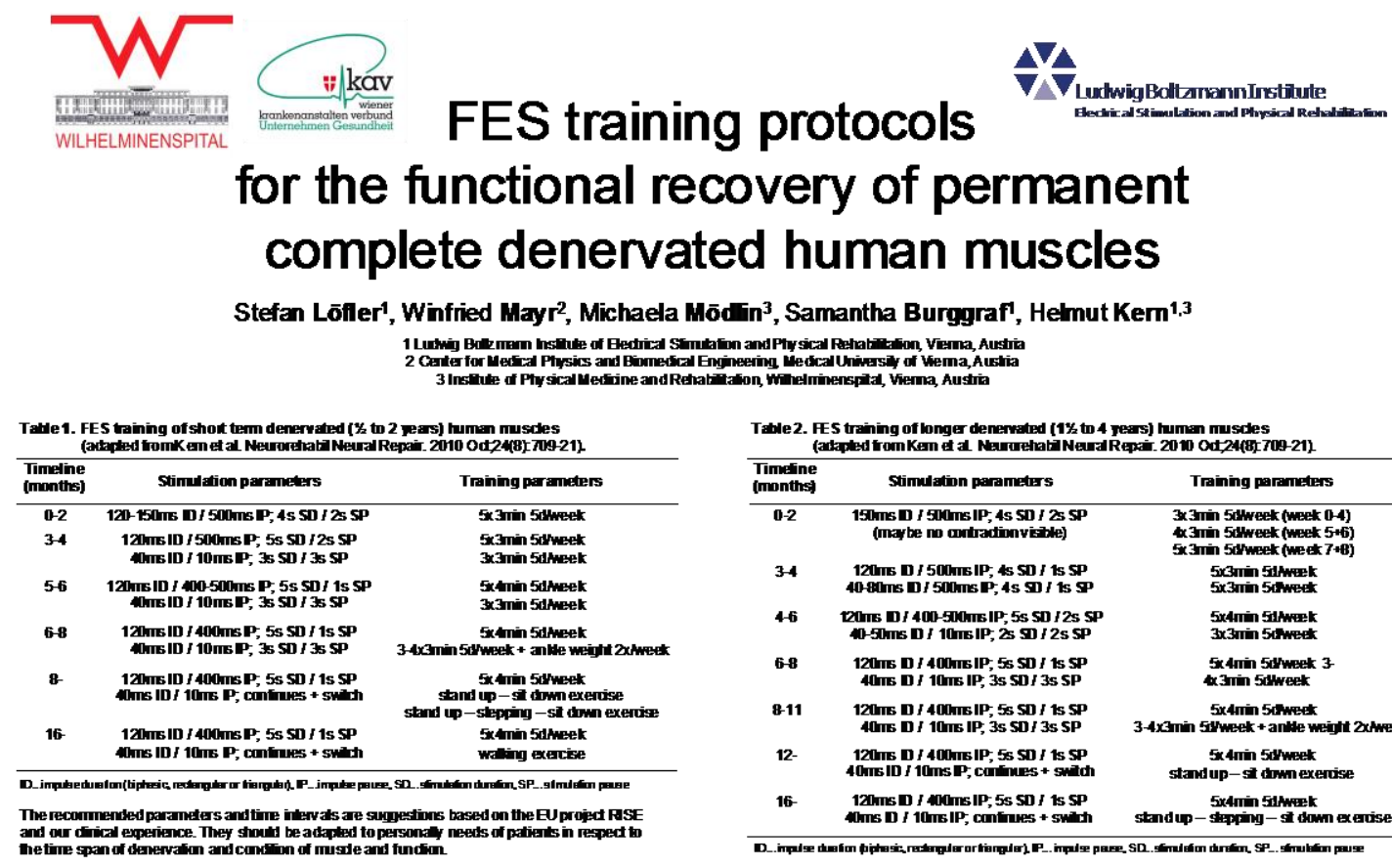

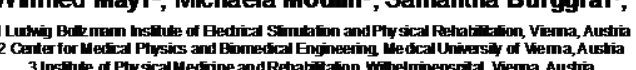
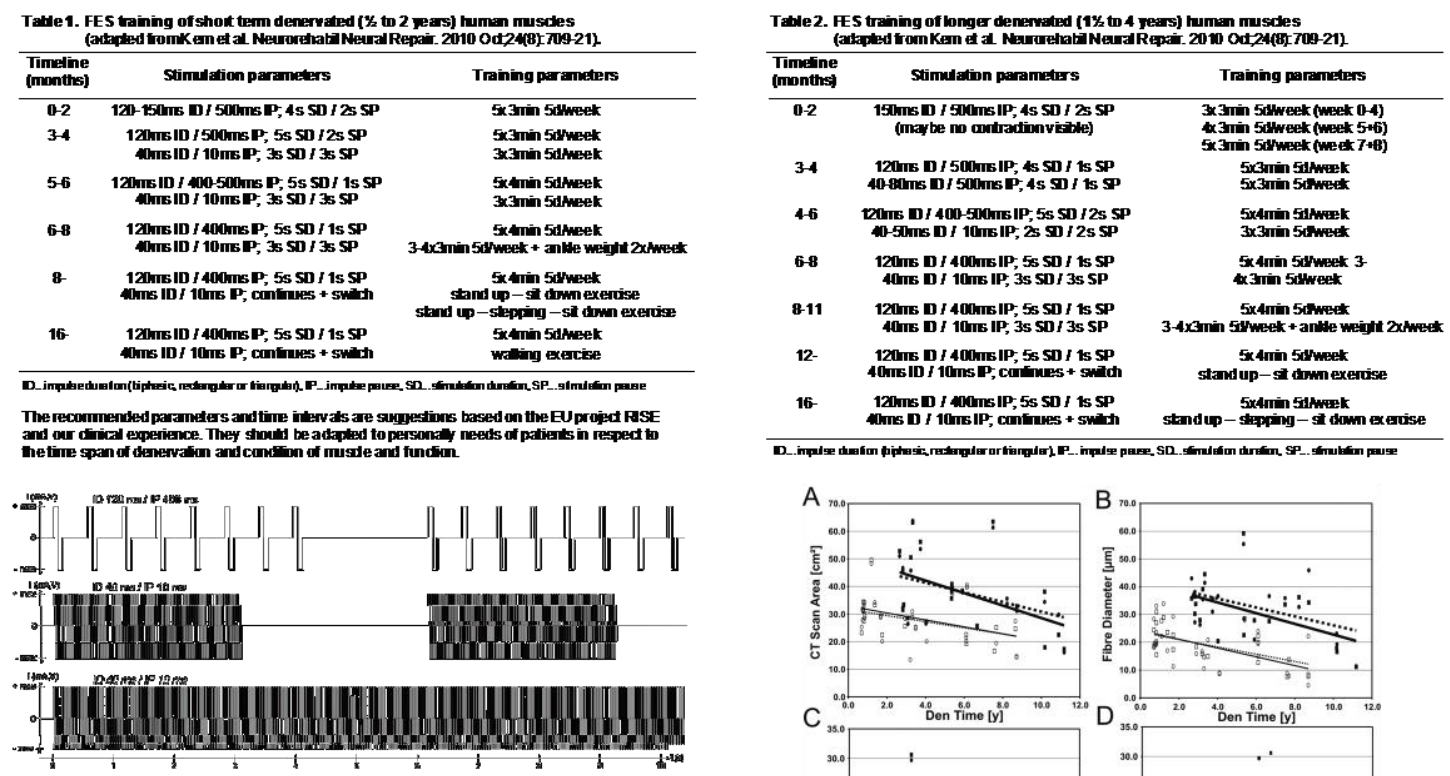

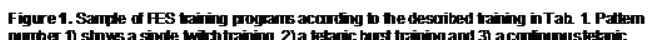

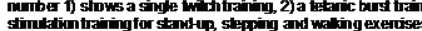

Sample of an progressive tunctional electrical stimudation (FES) training accodting to the described training in the Tah. 1 and showatso in Fig. 16.7.

- It ctats with busts of a simtatim dratim (SD) of ts and a stimution pausP (SP) of $2 \mathrm{~S}$

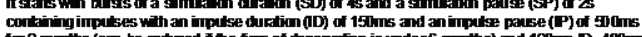

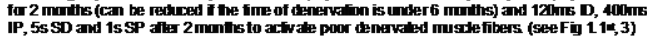

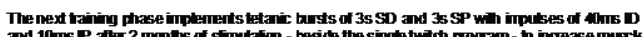

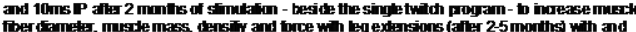

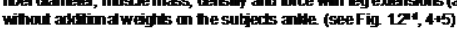

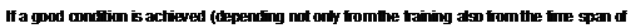

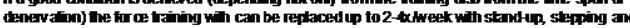

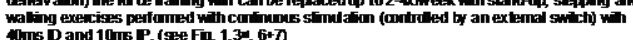

\begin{tabular}{|c|c|c|}
\hline $\begin{array}{l}\text { Timaline } \\
\text { (months) }\end{array}$ & Stimulation parametes & Training parameters \\
\hline $0-2$ & 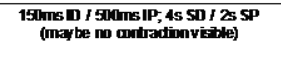 & 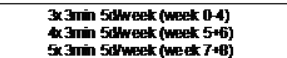 \\
\hline 34 & 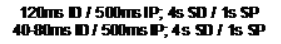 & 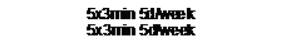 \\
\hline 46 & 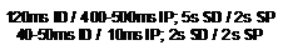 & 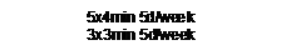 \\
\hline 68 & 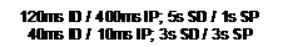 & $\begin{array}{l}\text { Sx Amin 5idweek } 3 \\
\text { 4x 3min 5ilweek }\end{array}$ \\
\hline $8-11$ & 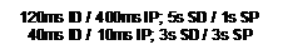 & 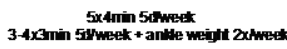 \\
\hline 12- & 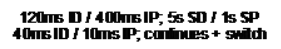 & 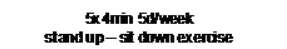 \\
\hline 16- & 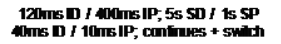 & 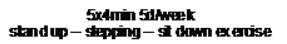 \\
\hline
\end{tabular}
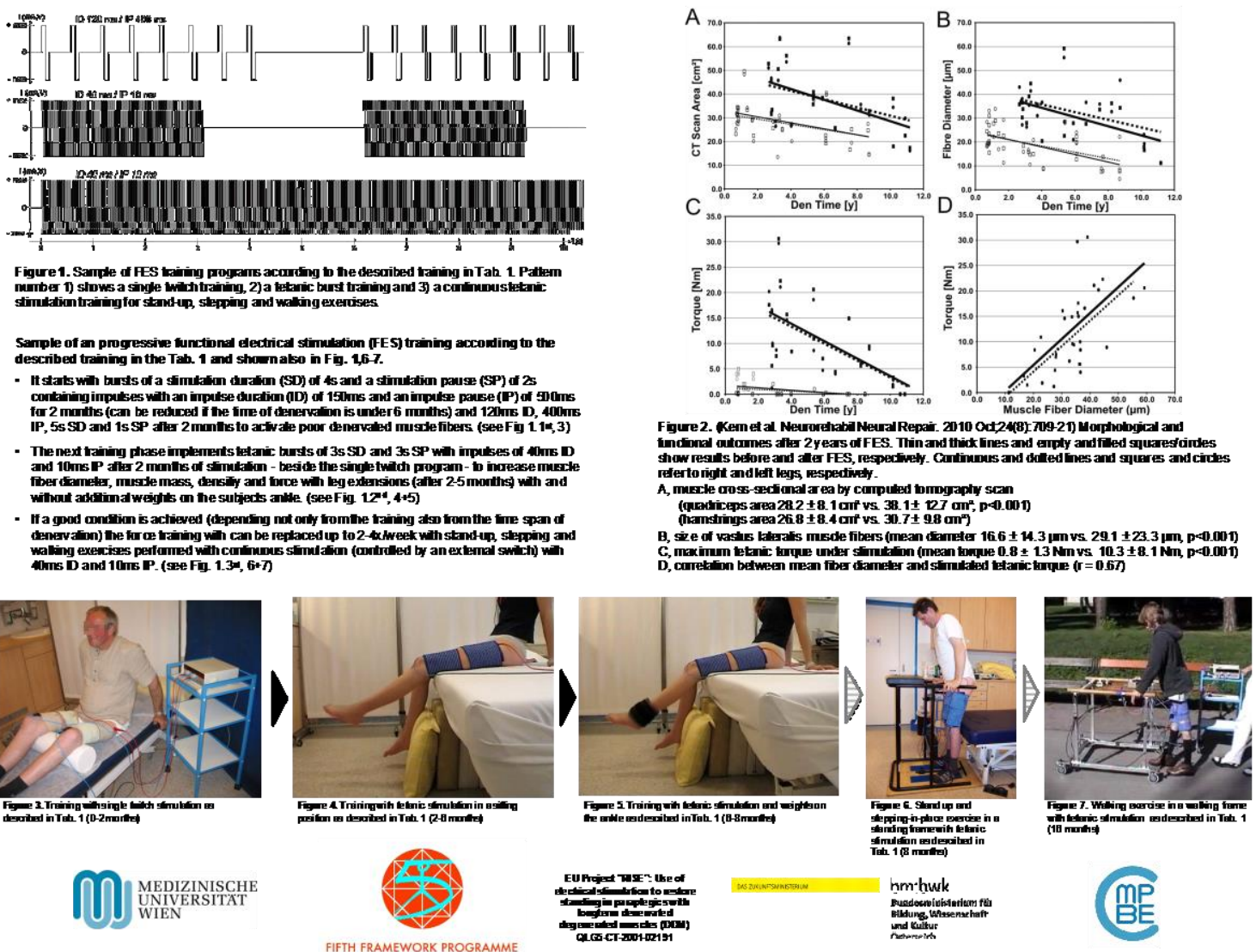

Figure2. Kemet al New Time (y)

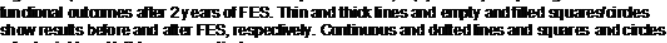
refertoright andleft legs espedivis.

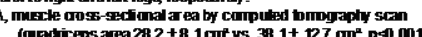

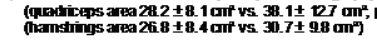

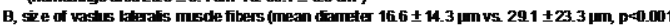

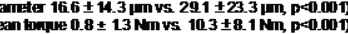

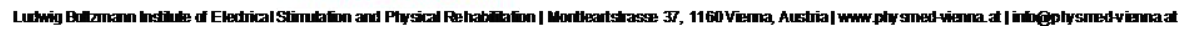

Figure 1. Vienna progressive h-b FES strategy for long-term complete denervation of quadriceps muscles

the necessary high current density, gel was used under the polyurethane electrodes to achieve minimal transition impedance. A special design feature was a non conductive bulge along the entire edge of the electrode that prevents potential skin burns that presumably can occur where a conductive edge gets in electrical contact with skin surface and causes local current density hot-spots (Mayr W, 2007; Patent - 


\section{Home-based FES for long-term denervated human muscle}

Eur J Trans Myol - Basic Appl Myol 2014; 24 (1): 27-40

Surface Electrode, EP2021068, WO/2007/131248). The electrodes were flexible enough to maintain evenly distributed pressure to the uneven and moving skin, thus providing homogeneous current distribution throughout the entire contact area.

Stimulation needle electromyography (SNMEG) was used to study the electrophysiological properties of single muscle fibers. We measured the muscle fiber conduction velocity (MFCV) and the shortest interstimulus interval (ISI) still eliciting a response to the second stimulus delivered to the fiber. ${ }^{76} \mathrm{MFCV}$ recorded in the denervated patients before and after h-b FES therapy showed a significant increase in conduction velocity (fastest and mean $\mathrm{CV}$ ) and reduced refractory periods (shortest ISI). This suggests that electrical stimulation training is effective to improve the electrical properties of the muscle fibre and SNEMG could serve as an additional measurement technique to specify the status of the denervated muscle. The training strategy consisted of two combined stimulation programs. ${ }^{31,38}$ All applicable rules concerning the ethical use of human volunteers were followed during the course of this research (Approval of Ethical Committee, Vienna, Austria: EK02-068-0702). For a multilingual translation of the Work Packages, ethical and safety issues link to: http://www.bio.unipd.it/bam/bam18-2\&3.html

At the beginning of the treatment, biphasic stimulation impulses of very long-duration (120-150 ms, 60-75 ms per phase) at high intensity (up to $\pm 80 \mathrm{~V}$ and up to \pm 250 $\mathrm{mA}$ ) were applied (Training Program 1). Then the subjects underwent clinical assessment and stimulated knee torque measurement every 12 weeks by physiatrists, who progressively modified the stimulation. The routine daily training consisted of combined twitch and tetanic stimulation patterns (Training Programs 2, 3 and 4) in consecutive sessions lasting up to $30 \mathrm{~min}$ for each group of muscles (gluteus, thigh and lower leg muscles on both sides). After tetanic contractility was achieved and the subject achieved full extension of the leg, the ankle was progressively loaded (Figure 1). Finally, the more compliant patients became able to stand and perform step-in-place and (if young and light) walking exercise. $^{37,38}$

\section{Perspectives}

In collaboration with his international partners, Dr. Kern is now extending the benefits of h-b FES to those subjects, which for different reasons, from the mild but unrelenting process of aging to the devastating fast progression of muscle atrophy in cancer patients, suffer the consequences of muscle weakness. Further, a multi-disciplinary research team of the Interdepartmental Research Center of Myology of the University of Padua is applying the Vienna principles to the apparently easier cases of peripheral incomplete denervation of arms and legs.
Examples in literature of the effectiveness of life-long high-level physical activity in postponing effects of aging, ${ }^{3,4,77}$ and of physical approaches in peripheral and central neural repair, seems to open new perspectives to an approach, home based Functional Electrical Stimulation in paraplegics by implanted electrodes and neuromodulators, that has been abandoned twenty or more years ago despite the successes of heart pacing and mini-implants for deaf, two very successful cases of Functional Electrical Stimulation of human tissues. $\mathrm{H}-\mathrm{b}$ FES is worth to be reassessed under strict scientific rules, balancing its costs against the needs and rights of patients to see alleviated their burdens.

\section{Financial support}

EU Commission Shared Cost Project RISE (Contract n. QLG5-CT-2001-02191) and The Austrian Ministry of Science funds to Prof. DDr. H. Kern and Prof. DI DDr. W. Mayr, Vienna (Austria) covered the clinical costs, the production of customized devices and the international management of the project. Italian MIUR and Telethon Grant GGP08153 funds to Prof. F. Protasi, CeSI, Chieti, Italy supported EM analyses. Italian MIUR funds to the Laboratory of Translational Myology, and Italian C.N.R. funds to the Institute of Neuroscience, University of Padova, Italy, supported light microscopy, morphometry, and costs of data analyses

\section{Acknowledgements}

This paper is dedicated to Herwig Thoma, without his creativity and his Vienna FES Meetings, neither the Vienna-Padua collaborations nor the Padua Muscle Days would have existed, nothing to say, the European Journal of Translational Myology.

We thank Gerta Vrbova for critical reading of the manuscript and for permission to quote her thoughtful observations on limitations of electrical stimulation of (denervated) muscles.

Authors of this article are indebted with all Partners of the European Project RISE: Use of electrical stimulation to restore standing in paraplegics with long-term denervated degenerated muscles (Contract no. QLG5-CT-2001-02191): M. Bijak and E. Unger, Biomedical Technology Center, Vienna, Austria; H. A. Cerrel Bazo, Neuromotor Rehabilitation, Cernusco, Milan, Italy; M. R. Dimitrijevic, Physical Medicine and Rehabilitation, Baylor College of Medicine, Houston, TX, USA; G. Exner, Spinal Cord Injury Center, Hamburg, Germany; E. Gallasch, Physiology, Graz, Austria; H. J. Gerner and R. Rupp, Orthopedics, Heidelberg, Germany; W. Girsch, Orthopedics, Speising, Vienna, Austria; T. Helgason, P. Ingvarsson, and S. Yngvason, Landspitali-University Hospital, Reykjavik, Iceland; J. Hufgard and M. Obrovsky, Rehabilitation, Klosterneuburg, Austria; H. P. Jonas, Rehabilitation, Bad Häring, Tirol, Austria; S. Lotta, Villanova sull'Arda (PC), Italy; D. Maier and M. 


\section{Home-based FES for long-term denervated human muscle}

Eur J Trans Myol - Basic Appl Myol 2014; 24 (1): 27-40

Potulski, Murnau, Spinal Cord Injury, Murnau, Germany; D. Rafolt, Institut für Biomedizinische Technik und Physik, Vienna, Austria.

We are also indebted with all the collaborators and the coauthors of the papers reporting RISE and Mobility in Aging results published from 2002 to date: Abruzzo PM, Adami N, Barberi L, Bassetto F, Biral D, Boato N, Boncompagni S, Bosco G, Burggraf S, Coletto L, Corbianco S, Cvecka J, Danieli-Betto D, De Rossi M, di Tullio S, Doria A, Fanò G, Ferrero M, Forstner C, Francini F, Franz C, Fruhmann H, Fulle S, Gargiulo P, Germinario E, Grim-Stieger M, Hamar D, Helgason B, Helgason T, Hoellwarth U, Hofer C, Ingvarsson P, Kovarik J, Krenn M, La Rovere R, Lapalombella R, Löfler S, Mancinelli R, Marcante A, Marini M, Masiero S, Mayr W, Merigliano S, Mödlin M, Mosole S, Musarò A, Nori A, Pond A, Paolini C, PaternostroSluga T, Pelosi L, Pietrangelo L, Pietrangelo T, Podhorska-Okolow M, Pond A, Protasi F, Rampudda ME, Reynisson PJ, Romanello V, Rossini K, Rupp R, Salmons S, Sandri M, Sarabon N, Sarzo G, Scordari A, Sedliak M, Squecco R, Stramare R, Tirpáková V, Trimmel L, Valente M, Vecchiato M, Vindigni V, Vogelauer M, Zampieri S, Zanato R, Zanin ME

\section{Corresponding Author}

Ugo Carraro, CIR-Myo Translational Myology Lab, Department of Biomedical Sciences, University of Padova, Italy

E-mail: ugo.carraro@unipd.it

\section{References}

1. Gutmann E, ed. The denervated muscle. Pub. House of the Czechoslovak Academy of Sciences, 1962.

2. Carraro U. Alterazioni delle funzioni delle membrane substrutturali nel muscolo denervato. M.D. Thesis, School of Medicine, University of Padova (Italy), pp.1-51, 1968.

3. Mosole S, Rossini K, Kern H,et al. Reinnervation of Vastus lateralis is increased significantly in older men (70-years old) with a lifelong history of high-level exercise. Eur J Trans Myol - Basic Appl Myol 2013;23:205-10.

4. Mosole S, Carraro U, Kern H, et al. Long term high-level exercise promotes muscle reinnervation with age. J Neuropathol Exp Neurol 2014,73, in press.

5. Carraro U, Catani C, Biral D. Selective maintenance of neurotrophically regulated proteins in denervated rat diaphragm. Exp Neurol 1979;63:468-75.

6. Carraro U, Catani C. A sensitive SDS-PAGE method separating myosin heavy chain isoforms of rat skeletal muscles reveals the heterogeneous nature of the embryonic myosin. Biochem Biophys Res Commun 1983;116:793-802.
7. Rossini K, Rizzi C, Sandri M, Carraro U. Highresolution sodium dodecyl sulfate-polyacrylamide gel electrophoresis and immunochemical identification of the $2 \mathrm{X}$ and embryonic myosin heavy chains in complex mixtures of isomyosins. Electrophoresis 1995;16:101-4.

8. Margreth A, Salviati G, Carraro U. Neural control on the activity of the calcium-transport system in sarcoplasmic reticulum of rat skeletal muscle. Nature 1973;24:285-6.

9. Carraro U, Morale D, Mussini I, et al. Chronic denervation of rat hemidiaphragm: maintenance of fiber heterogeneity with associated increasing uniformity of myosin isoforms. J Cell Biol 1985;100:161-74.

10. Lømo T, Rosenthal J. Control of ACh sensitivity by muscle activity in the rat. $\mathrm{J}$ Physiol 1972;221:493-513.

11. Lømo T, Westgaard RH, Dahl HA. Contractile properties of muscle: control by pattern of muscle activity in the rat. Proc R Soc Lond, B, Biol Sci 1974:187:99-103.

12. Lømo T. Neurotrophic control of colchicine effects on muscle? Nature 1974;249:473-4.

13. Lømo T, Westgaard RH. Further studies on the control of ACh sensitivity by muscle activity in the rat. J Physiol 1975;252, 603-26.

14. Westgaard RH. Influence of activity on the passive electrical properties of denervated soleus muscle fibres in the rat. J Physiol 1975;251:68397.

15. Lømo T, Westgaard RH. Control of ACh sensitivity in rat muscle fibers. Cold Spring Harbor symposia on quantitative biology 1976;40,263-74

16. Westgaard RH, Lømo T. Control of contractile properties within adaptive ranges by patterns of impulse activity in the rat. J Neurosci 1988:8, 4415-26.

17. Eken T, Gundersen K. Electrical stimulation resembling normal motor-unit activity: effects on denervated fast and slow rat muscles. J Physiol 1988;402:651-69.

18. Ausoni S, Gorza L, Schiaffino S, Gundersen K, Lømo T. Expression of myosin heavy chain isoforms in stimulated fast and slow rat muscles. J Neurosci 1990;10:153-60.

19 Schiaffino S, Reggiani C. Myosin isoforms in mammalian skeletal muscle. J Appl Physiol (1985). 1994;77(2):493-501.

20. Carraro U, Catani C, Belluco S, et al. Slow-like electrostimulation switches on slow myosin in denervated fast muscle. Exp Neurol 1986;94:53753.

21. Mussini I, Favaro G, Carraro U. Maturation, dystrophic changes and the continuous production of fibers in skeletal muscle regenerating in the 


\section{Home-based FES for long-term denervated human muscle}

Eur J Trans Myol - Basic Appl Myol 2014; 24 (1): 27-40

absence of nerve. J Neuropathol Exp Neurol 1987;46:315-31.

22. Cantini M, Carraro U. Macrophage-released factor stimulates selectively myogenic cells in primary muscle culture. J Neuropathol Exp Neurol 1995;54:121-8.

23. Sandri M, Carraro U, Podhorska-Okolov M, et al. Apoptosis, DNA damage and ubiquitin expression in normal and mdx muscle fibers after exercise. FEBS Lett 1995;373:291-25.

24. Sandri M, Minetti C, Pedemonte M, Carraro U. Apoptotic myonuclei in human Duchenne muscular dystrophy. Lab Invest 1998;78:1005-16.

25. Vescovo G, Serafini F, Facchin L, et al. Specific changes in skeletal muscle myosin heavy chain composition in cardiac failure: differences compared with disuse atrophy as assessed on microbiopsies by high resolution electrophoresis. Heart 1996;76:337-43.

26. Arpesella G, Carraro U, Mikus PM, et al. Activity-rest stimulation of latissimus dorsi for cardiomyoplasty: 1-year results in sheep. Ann Thorac Surg 1998;66:1983-90.

27. Rigatelli G, Carraro U, Barbiero $M$, et al. Activity-rest stimulation protocol improves cardiac assistance in dynamic cardiomyoplasty. Eur J Cardiothorac Surg 2002;21:478-82.

28. Carraro U, Catani C, Saggin L, et al. Isomyosin changes after functional electrostimulation of denervated sheep muscle. Muscle Nerve 1988;11:1016-28.

29. Ludlow CL, Bielamowicz S, Daniels Rosenberg $\mathrm{M}$, et al. Chronic intermittent stimulation of the thyroarytenoid muscle maintains dynamic control of glottal adduction. Muscle Nerve 2000;23:4457.

30. Rossini K, Zanin ME, Carraro U. To stage and quantify regenerative myogenesis in human longterm permanent denervated muscle. Basic Appl Myol 2002;12:277-86.

31. Kern H, Boncompagni S, Rossini K, et al. Longterm denervation in humans causes degeneration of both contractile and excitation-contraction coupling apparatus, which is reversible by functional electrical stimulation (FES): a role for myofiber regeneration? J Neuropathol Exp Neurol 2004;63:919-31.

32. Kern H, Salmons S, Mayr W, Rossini K, Carraro U. Recovery of long-term denervated human muscles induced by electrical stimulation. Muscle Nerve 2005;31:98-101.

33. Carraro U, Rossini K, Mayr W, Kern H. Muscle fiber regeneration in human permanent lower motoneuron denervation: relevance to safety and effectiveness of FES-training, which induces muscle recovery in SCI subjects. Artif Organs 2005;29:187-91.
34. Boncompagni S, Kern $\mathrm{H}$, Rossini $\mathrm{K}$, et al. Structural differentiation of skeletal muscle fibers in the absence of innervation in humans. Proc Natl Acad Sci U S A 2007;104:19339-44.

35. Kern H, Hofer C, Mayr W, Carraro U. European Project RISE: Partners, protocols, demography. Basic Appl Myol/ Eur J Trans Myol 2009;19:2116.

36. Squecco R, Carraro U, Kern H, et al. A subpopulation of rat muscle fibers maintains an assessable excitation-contraction coupling mechanism after long-standing denervation despite lost contractility. J Neuropathol Exp Neurol 2009;68:1256-68.

37. Kern H, Carraro U, Adami N, et al. One year of home-based daily FES in complete lower motor neuron paraplegia: recovery of tetanic contractility drives the structural improvements of denervated muscle. Neurol Res 2010;32:5-12.

38. Kern H, Carraro U, Adami N, et al. Home-based functional electrical stimulation rescues permanently denervated muscles in paraplegic patients with complete lower motor neuron lesion. Neurorehabil Neural Repair 2010;24:70921.

39. Gargiulo P, Helgason T, Reynisson PJ, et al. Monitoring of muscle and bone recovery in spinal cord injury patients treated with electrical stimulation using three-dimensional imaging and segmentation techniques: methodological assessment. Artif Organs 2011;35:275-81.

40. Mancinelli $\mathrm{R}$, Kern $\mathrm{H}$, Fulle $\mathrm{S}$, et al. Transcriptional profile of denervated vastus lateralis muscle derived from a patient 8 months after spinal cord injury: a case-report. Int $\mathbf{J}$ Immunopathol Pharmacol 2011;24:749-59.

41. Kern H, Pelosi L, Coletto L, et al. Atrophy/hypertrophy cell signaling in muscles of young athletes trained with vibrationalproprioceptive stimulation. Neurol Res 2011;33:998-1009.

42. Kern H, Kovarik J, Franz C, et al. Effects of 8 weeks of vibration training at different frequencies ( 1 or $15 \mathrm{~Hz}$ ) in senior sportsmen on torque and force development and of 1 year of training on muscle fibers. Neurol Res 2010;32:26-31.

43. Zampieri S, Valente $\mathrm{M}$, Adami $\mathrm{N}$, et al. Polymyositis, dermatomyositis and malignancy: a further intriguing link. Autoimmun Rev 2010;9:449-53.

44. Marcante A, Zanato R, Ferrero M, et al. Recovery of tetanic contractility of denervated muscle: A step toward a walking aid for foot drop. Biomed Tech (Berl) 2013 Sep 7. doi:pii: /j/bmte.2013.58.issue-s1-A/bmt-2013-4016/bmt2013-4016.xml. 10.1515/bmt-2013-4016. [Epub ahead of print] 


\section{Home-based FES for long-term denervated human muscle}

Eur J Trans Myol - Basic Appl Myol 2014; 24 (1): 27-40

45. Zanato R, Stramare R, Boato N, et al. Dynamic Echomyography shows that FES in peripheral denervation does not hamper muscle reinnervation. Biomed Tech (Berl) 2013 Sep 7. doi:pii: /j/bmte.2013.58.issue-s1-A/bmt-20134034/bmt-2013-4034.xml. 10.1515/bmt-20134034. [Epub ahead of print]

46. Gargiulo P, Helgason T, Ramon C, et al. CT and MRI assessment and characterization using segmentation techniques and 3D modeling technique: a review on applications to muscle, bone and brain tissues. Eur J Trans Myol - Basic Appl Myol 2014;24:55-63.

47. Kern H. Funktionelle Elektrostimulation paraplegischer patienten. Österr Z Phys Med. 1995;5:1-79.

48. Kern H. Habilitation Thesis Functional Electrical Stimulation on Paraplegic Patients. Eur J Trans Myol - Basic Appl Myol 2014;24:in press.

49. Holle J, Frey M, Gruber H, et al. Functional electrostimulation of paraplegics (experimental investigations and first clinical experience with an implantable stimulation device). J Orthopedics 1984;7:1145-55

50. Carlson B. The biology of long-term denervated skeletal muscle. Eur J Trans Myol - Basic Appl Myol 2014;24:5-11.

51. Carlson BM. The Denervated Muscle: 45 years later. Neurol Res 2008;30:119-22.

52. Kauhanen MS, Lorenzetti F, Leivo IV, et al. Long-term morphometric and immunohistochemical findings in human free microvascular muscle flaps. Microsurgery. 2004;24:30-8.

53. Ashley Z, Sutherland $\mathrm{H}$, Lanmüller $\mathrm{H}$, et al. Atrophy, but not necrosis, in rabbit skeletal muscle denervated for periods up to one year. Am J Physiol Cell Physiol 2007;292:440-51.

54. Kern H, Carraro U, Biral D, et al. Severely atrophic muscle fibers with nuclear clumps survive many years in permanently denervated human muscle. The Open Pathology Journal 2009;3:106-10.

55. Carraro U. Modulation of trophism and fiber type expression of denervated muscle by different patterns of electrical stimulation. Basic Appl Myol 2002;12:263-72.

56. Adams L, Carlson BM, Henderson L, Goldman D. Adaptation of nicotinic acetylcholine receptor, myogenin, and MRF4 gene expression to longterm muscle denervation. $\mathrm{J}$ Cell Biol 1995;131:1341-49.

57 Lapalombella R, Kern H, Adami N, et al. Persistence of regenerative myogenesis in spite of down-regulation of activity-dependent genes in long-term denervated rat muscle. Neurol Res 2008;30:197-206.
58. Kern H, Rossini K, Carraro U, et al. Muscle biopsies show that FES of denervated muscles reverses human muscle degeneration from permanent spinal motoneuron lesion. J Rehabil Res Dev 2005;42:43-53.

59. Kern H, Hofer C, Mödlin M, et al. Stable muscle atrophy in long-term paraplegics with complete upper motor neuron lesion from 3- to 20-year SCI. Spinal Cord 2008;46:293-304.

60. Kern H, Hofer C, Mayr W, Carraro U. European Project RISE: Partners, protocols, demography. Basic Appl Myol 2009;19:211-6.

61. Kern H, Hofer C, Mayr W. Protocols for Clinical Work Package of the European Project RISE. Basic Appl Myol 2008;18:39-44.

62. Sunderland S. Nerve and Nerve Injuries. 2nd ed. Edinburgh, UK: Churchill-Livingston; 1978.

63. Harrison D. Current trends in the treatment of established unilateral facial palsy. In: Karcher E, ed. Functional Surgery of the Head and Neck. Proceedings of the first international meeting on Functional Surgery of the Head and Neck; Graz, Austria; 1989;9-16.

64. Kobayashi J, Mackinnon SE, Watanabe O, et al. The effect of duration of muscle denervation on functional recovery in the rat model. Muscle Nerve. 1997;20:858-66.

65. Nemoto K, Williams HB, Nemoto K, et al. The effects of electrical stimulation on denervated muscle using implantable electrodes. J Reconstr Microsurg. 1988;4:251-5.

66. Nightingale EJ, Raymond J, Middleton JW, et al. Benefits of FES gait in a spinal cord injured population. Spinal Cord. 2007;45:646-57.

67. Vrbova G. The effect of motoneurone activity on the speed of contraction of striated muscle. J Physiol. 1963;169:513-26.

68. Milner-Brown HS, Stein RB, Yemm R. The orderly recruitment of human motor units during voluntary isometric contraction. J Physiol 1973;230:359-70.

69. Mcminn RM, Vrbovà G. Motoneurone activity as a cause of degeneration in the soleus muscle of the rabbit. Q J Exp Physiol Cogn Med Sci 1967;52:411-5.

70. Kern H, Hofer Ch, Mayr W, et al. Elektrostimulation komplett denervierter Muskulatur. In Fialka-Moser V (Ed.), Kompendium der Physikalischen Medizin und Rehabilitation (3rd ed.), 2012, pp. 445-456. Vienna: Springer Verlag.

71. Hofer C, Mayr W, Stöhr H, et al. A stimulator for functional activation of denervated muscles. Artif Organs 2002;26:276-9.

72. Mayr W, Bijak M, Rafolt D, Sauermann S, Unger E, Lanmüller H. Basic design and construction of the Vienna FES implants: existing solutions and 


\title{
Home-based FES for long-term denervated human muscle
}

\author{
Eur J Trans Myol - Basic Appl Myol 2014; 24 (1): 27-40
}

prospects for new generations of implants. Med Eng Phys 2001;23:53-60.

73. Meyerspeer M, Mandl T, Reichel M, et al. Effects of functional electrical stimulation in denervated thigh muscles of paraplegic patients mapped with T2 imaging. MAGMA. 2008;21:219-26.

74. Kern H, Hofer C, Moedlin M, et al. Denervated muscle in humans: limitations and problems of currently used functional electrical stimulation training protocols. Artif Organs 2002;26:216-8.
75. Mödlin M, Forstner C, Hofer C, et al. Electrical stimulation of denervated muscles: first results of a clinical study. Artif Organs 2005;29:203-6.

76. Hofer C, Forstener C, Moedlin M, et al. In vivo assessment of conduction velocity and refractory period of denervatd muscle fibers. Artif Organs 2005;29:436-39.

77. Zampieri S, Pietrangelo L, Loefler S, et al. Lifelong Physical Exercise Delays AgeAssociated Skeletal Muscle Decline. J Gerontol A Biol Sci Med Sci 1 2014, doi:10.1093/gerona/glu006, in press. 